\title{
Características del crecimiento en diámetro, altura y volumen de una plantación de Eucalyptus nitens sometida a tratamientos silvícolas de poda y raleo
}

\author{
Growth characteristics in diameter, height, and volume of a Eucalyptus nitens plantation with \\ different silvicultural treatment for pruning and thinning \\ FERNANDO MUÑOZ ${ }^{1}$, MIGUEL ESPINOSA ${ }^{1}$, MIGUEL A. HERRERA ${ }^{2}$, JORGE CANCINO ${ }^{1}$ \\ ${ }^{1}$ Facultad de Ciencias Forestales, Universidad de Concepción, Casilla 160-C, Concepción, Chile. \\ E-mail: fmunoz@udec.cl \\ ${ }^{2}$ Escuela Técnica Superior de Ingenieros Agrónomos y de Montes, Universidad de Córdoba, \\ Apdo. 3048, Córdoba, España.
}

\begin{abstract}
SUMMARY
Chile has an important tree cover of Eucalyptus nitens (Deane and Maiden) plantations destined primarily for pulpwood production. Nevertheless, other products such as sawlogs and veneers are required because of the continually changing market for wood, as well as the need to obtain greater economic returns from the forest plantations. The present work describes the results obtained in a trial of pruning and thinning of Eucalyptus nitens plantations during eight years of control. The trial was established in 1989 and was located close to Los Alamos, Eighth Region, Chile, and involved observing an arrangement of two factors: intensity of thinning (without thinning, and thinning to 800 and 400 trees $\mathrm{ha}^{-1}$ ) and severity of pruning (without pruning, and pruning at 3.5 and $7.0 \mathrm{~m}$ ), and three replications. Three measurements were taken: at the establishment of the trial, in October 1995 (6 years old), in October 1999 (10 years old), and in 2003 (14 years old). The diameter at breast height (dbh) was measured for all the trees of the plots, and the total height in a subsample of 15 trees per plot. After eight years of growth, the plots most intensely thinned reached the greatest tree growth in diameter and volume. Thinning has a significant effect in growth, but not pruning. Growth in diameter, height, and volume by tree of the 400 trees per hectare of greater dbh did not vary significantly with the treatment. The greatest volume per hectare was gained with 1100 trees ha ${ }^{-1}$ and $3.5 \mathrm{~m}$ pruning, with $680.0 \mathrm{~m}^{3} \mathrm{ha}^{-1}$ and a mean annual increment (MAI) of $48.6 \mathrm{~m}^{3} \mathrm{ha}^{-1}$ year ${ }^{-1}$.
\end{abstract}

Keywords: pruning, thinning, plantation, Eucalyptus nitens.

\section{RESUMEN}

Chile posee una importante superficie de plantaciones de Eucalyptus nitens (Deane \& Maiden) Maiden destinada fundamentalmente a la obtención de madera para pulpa. Sin embargo, debido a los permanentes cambios que presenta el mercado de la madera y la necesidad de obtener mayores retornos económicos de los cultivos forestales, se requiere obtener otros productos provenientes de estas plantaciones, como trozas para madera aserrada y chapas. El presente documento entrega los resultados obtenidos en un ensayo de poda y raleo en Eucalyptus nitens después de ocho años de control. El ensayo se estableció en una plantación del año 1989, ubicada cercana a la localidad de Los Alamos, VIII Región, según un arreglo factorial de dos factores: intensidad de raleo (sin raleo, 800 y $400 \mathrm{arb} \mathrm{ha}^{-1}$ ) y severidad de poda (sin poda, 3,5 y 7,0 m) y tres repeticiones. Se efectuaron tres mediciones: al establecimiento del ensayo, en octubre de 1995 (6 años de edad), y posteriormente en octubre de los años 1999 (10 años de edad) y 2003 (14 años de edad). Se midió el diámetro a la altura del pecho (dap) de todos los árboles y alturas totales en una submuestra de 15 árboles por parcela. Ocho años después de aplicado los tratamientos, las parcelas más intensamente raleadas presentan los mayores crecimientos en diámetro y volumen por árbol. El raleo tuvo un efecto significativo en el crecimiento de los árboles, no así la poda. El crecimiento en diámetro, altura y volumen por árbol de los 400 árboles de mayor dap por hectárea no varía significativamente entre tratamientos. El mayor volumen por hectárea se alcanza en el tratamiento 1.100 árb $\mathrm{ha}^{-1}$ y $3,5 \mathrm{~m}$ de poda con $680,0 \mathrm{~m}^{3} \mathrm{ha}^{-1}$ y un incremento medio anual (IMA) de $48,6 \mathrm{~m}^{3} \mathrm{ha}^{-1} \mathrm{año}^{-1}$.

Palabras clave: poda, raleo, plantación, Eucalyptus nitens. 


\section{INTRODUCCION}

El establecimiento de plantaciones de Eucalyptus nitens (Deane \& Maiden) Maiden constituye una nueva alternativa para la creación de bosques con una especie de rápido crecimiento y tolerante a un amplio rango de condiciones ambientales (1, 2). La superficie mundial de plantaciones con esta especie es de aproximadamente 340.000 ha, distribuidas en Australia (Tasmania y Victoria), Chile, Sudáfrica, Nueva Zelanda y España, la mayor parte de las cuales (88\%) en Australia y Chile (3). En ambos países se utiliza mayoritariamente para pulpa $(3,4,5)$.

En Australia (Tasmania), desde hace algunos años se realizan esfuerzos para incorporar podas en el manejo de las plantaciones de E. nitens, que permitan generar madera de mayor calidad (6). En Chile, el manejo de E. nitens no es una práctica frecuente y son escasos los antecedentes publicados sobre esquemas de manejo orientados a la producción de manera aserrada (3). Stöckle (7) y Muñoz y Espinosa (8), evaluaron los resultados preliminares de un ensayo de poda y raleo en un rodal de E. nitens de 6 años de edad, luego de uno y tres años de establecido, respectivamente. Celhay et al. (9) determinaron el efecto de diferentes severidades de poda en plantaciones de esta especie luego de 22 meses de establecido el experimento.

Eucalyptus nitens es una especie que no presenta poda natural, lo que genera la presencia de nudos en la madera $(3,4)$. Por consiguiente, la poda de ramas antes de su muerte es considerada esencial para la producción de madera de alta calidad $(1,10)$. A su vez, la combinación de regímenes de poda y raleo aplicado al manejo de esta especie, permite controlar la dimensión de los nudos, limitando este defecto a una porción reducida del corazón nudoso del fuste (11).

El objetivo de este estudio es evaluar el efecto de diferentes tratamientos de poda y raleo en el crecimiento en diámetro, altura y volumen de una plantación de E. nitens de 14 años de edad, luego de 8 años de establecido el experimento.

\section{MATERIAL Y METODOS}

El ensayo se estableció en la localidad de Los Alamos ( $37^{\circ} 38^{\prime}$ latitud sur, $73^{\circ} 27^{\prime}$ longitud oes- te), provincia de Arauco, Octava Región, en un rodal de E. nitens de 4,2 ha de superficie y altitud de 179,6 ms.n.m. La topografía es de lomaje suave y el suelo originado a partir de sedimentos marinos. Son suelos de textura franco arcillosa, ligeramente plásticos y adhesivos en húmedo, duros y compactos en seco. Presenta una fase de erosión fuerte y otra muy erosionada (12). Las características climáticas generales de la zona varían de una máxima en el mes de enero de 23,2 ${ }^{\circ} \mathrm{C}$ y una mínima en junio de $6,0^{\circ} \mathrm{C}$; el período libre de heladas es de 322 días, con un promedio de una helada por año y 28 días de frío. La precipitación promedio anual es de $1.437 \mathrm{~mm}$ con un período seco de cinco meses (13).

La plantación se realizó en invierno de 1989 con un espaciamiento de 2,0 x 3,5 m, sobre líneas de subsolado, con plantas producidas de semillas de procedencia Toorongo (Victoria, Australia). Previo a la plantación se efectuó control de malezas. El cultivo anterior fue una plantación de pino radiata (Pinus radiata D. Don), cuyos residuos generados por la cosecha fueron quemados.

Los tratamientos resultan de la combinación de tres densidades nominales de rodal (1.100 ( $\sin$

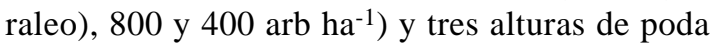
(0 (sin poda), 3,5 y 7,0 m). Por cada tratamiento ( 9 en total) se realizaron tres repeticiones, con un total de 27 parcelas, a las que se les asignaron los tratamientos en forma aleatoria. Las parcelas son cuadradas de $324 \mathrm{~m}^{2}(18 \times 18 \mathrm{~m})$, rodeadas por una faja perimetral de aislamiento de $5 \mathrm{~m}$ de ancho.

Las intervenciones de poda y raleo se efectuaron en octubre de 1995, cuando el rodal tenía seis años de edad. Se efectuaron tres mediciones: al establecimiento del ensayo, en octubre de 1995, y posteriormente en octubre del año 1999 (10 años de edad) y octubre del año 2003 (14 años de edad). Se midió el diámetro a la altura del pecho (dap) de todos los árboles y la altura total (alt) en una submuestra de 15 árboles por parcela, abarcando el espectro diamétrico registrado al inicio del estudio, submuestra que se mantuvo en todas las mediciones.

El diseño utilizado corresponde a un arreglo factorial de dos factores (altura de poda e intensidad de raleo) en tres niveles cada uno. El modelo estadístico es:

$$
y_{i j}=\mu+\tau_{i}+\beta_{j}+(\tau \beta)_{i j}+\varepsilon_{i j}
$$


donde,

$y_{i j}=$ Representa la observación correspondiente al nivel $(i)$ del factor altura de poda y al nivel (j) del factor intensidad de raleo

$\mu=$ Efecto constante denominado media global

$\tau=$ Efecto producido por el nivel $i$-ésimo del factor altura de poda

$\beta=$ Efecto producido por el nivel $i$-ésimo del factor intensidad de raleo

$\tau \beta=$ Interacción entre el factor altura de poda e intensidad de raleo

$i$ = Niveles de altura de poda (0 (sin poda), 3,5 y 7,0 m de altura)

$j=$ Niveles de intensidad de raleo (1.100 ( $\sin$ raleo), 800 y 400 árb ha ${ }^{-1}$ )

$\varepsilon=$ Error aleatorio $\sim \mathrm{N}\left(0, \sigma_{\varepsilon}^{2}\right)$

Se evaluaron las variables dap, altura y volumen a través de análisis de varianza para determinar homogeneidad de datos al inicio del ensayo (1995). Todas las variables medidas en los controles efectuados (años 1995, 1999 y 2003) fueron sometidas a pruebas para determinar su normalidad y homogeneidad de varianzas. El análisis de datos recopilados al establecimiento del ensayo evidenció diferencias significativas en dap. Así, el dap se utilizó como covariable en el análisis de covarianza de la información de dap y volumen obtenida en las mediciones posteriores. El efecto de los factores principales se determinó mediante análisis de varianza (ANOVA) y las comparaciones a posteriori (post hoc) entre medias mediante la prueba de Tukey (14). Las diferencias fueron consideradas significativas con $\mathrm{p}<0,05$. Para el proceso de datos se utilizó el programa estadístico SPSS versión 12.0 para Windows. Se analizó el crecimiento en dap, altura y volumen entre controles sucesivos, considerando todos los árboles y también los 400 árb ha $^{-1}$ de mayor dap de cada tratamiento. La selección de árboles se realizó en base a la distribución diamétrica existente al final del periodo de medición (año 2003). El volumen se obtuvo utilizando una función de volumen individual para la especie con un diámetro límite de utilización de $5 \mathrm{~cm}$ (15).

\section{RESULTADOS}

Respuesta de las variables a los tratamientos: El crecimiento en dap, altura y volumen medio de los árboles incrementó en todos los tratamientos, particularmente en los espaciamientos más amplios. Al año 2003 (14 años de edad), oportunidad en que se efectuó el último control, el tratamiento de raleo más intenso (400 árb $\mathrm{ha}^{-1}$ ) presenta diferencias significativas con respecto a los restantes tratamientos de raleo, variables dap y vol, independiente del nivel de poda aplicado (cuadro 1).

A diferencia del raleo, la poda no tuvo un efecto significativo en el crecimiento de los árboles en todos los tratamientos (excepto variable vol a los 10 años), independientemente del nivel de raleo aplicado. De acuerdo al control realizado en el año 2003, para la misma intensidad de raleo, no hay diferencia significativa en los valores medios de dap, altura y volumen, entre los árboles de las parcelas más intensamente podadas ( 7 m) con los árboles sin poda (cuadro 1).

Respuesta de las variables en los 400 árboles de mayor dap por hectárea: Al año 2003, el crecimiento de los 400 árboles con mayor dap por hectárea no presenta diferencias significativas entre tratamientos. No hay efecto de los factores poda y raleo, como tampoco la interacción poda $\mathrm{x}$ raleo, en las variables medidas (cuadro 2). Igual comportamiento de las variables se determinó en el control del año 1999, cuando la plantación tenía 10 años de edad.

Crecimiento volumétrico por hectárea: Considerando todos los árboles del ensayo y de acuerdo al control del año 2003 (cuadro 3), el mayor incremento medio anual (IMA) se alcanza en los tratamientos con mayor densidad (T1, T4 y T7) con valores de 40,6, 48,6 y 46,6 $\mathrm{m}^{3} \mathrm{ha}^{-1} \mathrm{año}^{-1}$, respectivamente. La mayor tasa de aumento del IMA se registra entre los 6 y 10 años de edad de la plantación, con incrementos entre 12,0 y 24,3 $\mathrm{m}^{3}$ ha $^{-1}$ año ${ }^{-1}$, tasa de crecimiento que tiende a disminuir en el último control, alcanzando en el tratamiento $\mathrm{T} 7$ valores negativos $\left(-0,9 \mathrm{~m}^{3} \mathrm{ha}^{-1}\right.$ $\left.\mathrm{año}^{-1}\right)$. Al igual que el IMA, el incremento periódico anual (IPA) alcanza valores mayores entre los 6 y 10 años de la plantación (entre 30,0 y $60,7 \mathrm{~m}^{3} \mathrm{ha}^{-1} \mathrm{año}^{-1}$ ) para disminuir fuertemente en el período siguiente, alcanzando en todo los tratamientos valores negativos (entre -2,6 y -36,8 $\mathrm{m}^{3}$ ha $^{-1}$ año $\left.^{-1}\right)$. 


\section{CUADRO 1}

Dimensiones de los árboles según tratamiento, año de medición y edad de plantación.

Tree size according to treatment, year of measurement, and stand age.

\begin{tabular}{|c|c|c|c|c|c|c|c|c|c|c|c|}
\hline \multicolumn{3}{|c|}{ Tratamientos } & \multicolumn{3}{|c|}{ Medición 1995 (6 años) } & \multicolumn{3}{|c|}{ Medición 1999 (10 años) } & \multicolumn{3}{|c|}{ Medición 2003 (14 años) } \\
\hline Poda & Raleo & Cod & $\begin{array}{l}\text { Dap } \\
(\mathrm{cm})\end{array}$ & $\begin{array}{c}\text { Altura } \\
(\mathrm{m})\end{array}$ & $\begin{array}{l}\text { Volumen } \\
\left(\mathrm{m}^{3} \mathrm{arb}^{-1}\right)\end{array}$ & $\begin{array}{l}\text { Dap } \\
(\mathrm{cm})\end{array}$ & $\begin{array}{l}\text { Altura } \\
(\mathrm{m})\end{array}$ & $\begin{array}{l}\text { Volumen } \\
\left(\mathrm{m}^{3} \mathrm{arb}^{-1}\right)\end{array}$ & $\begin{array}{l}\text { Dap } \\
(\mathrm{cm})\end{array}$ & $\begin{array}{l}\text { Altura } \\
(\mathrm{m})\end{array}$ & $\begin{array}{l}\text { Volumen } \\
\left(\mathrm{m}^{3} \operatorname{arb}^{-1}\right)\end{array}$ \\
\hline $0 \mathrm{~m}$ & $\begin{array}{r}1.100 \\
800 \\
400\end{array}$ & $\begin{array}{l}\text { T1 } \\
\text { T2 } \\
\text { T3 }\end{array}$ & $\begin{array}{l}15,5 \mathrm{ab} \\
16,0 \mathrm{ab} \\
17,0 \mathrm{ab}\end{array}$ & $\begin{array}{l}18,1 \mathrm{a} \\
18,1 \mathrm{a} \\
17,8 \mathrm{a}\end{array}$ & $\begin{array}{l}0,133 \mathrm{a} \\
0,130 \mathrm{a} \\
0,148 \mathrm{a}\end{array}$ & $\begin{array}{l}20,8 \mathrm{~d} \\
23,0 \mathrm{bc} \\
27,0 \mathrm{a}\end{array}$ & $\begin{array}{l}26,2 \mathrm{a} \\
27,7 \mathrm{a} \\
28,9 \mathrm{a}\end{array}$ & $\begin{array}{l}0,357 \mathrm{~d} \\
0,448 \mathrm{bcd} \\
0,597 \mathrm{a}\end{array}$ & $\begin{array}{c}23,7 \mathrm{c} \\
26,3 \mathrm{bc} \\
32,3 \mathrm{a}\end{array}$ & $\begin{array}{l}28,7 \mathrm{~b} \\
30,5 \mathrm{ab} \\
33,6 \mathrm{a}\end{array}$ & $\begin{array}{l}0,507 \mathrm{~d} \\
0,652 \mathrm{~cd} \\
0,987 \mathrm{a}\end{array}$ \\
\hline $3,5 \mathrm{~m}$ & $\begin{array}{r}1.100 \\
800 \\
400\end{array}$ & $\begin{array}{l}\text { T4 } \\
\text { T5 } \\
\text { T6 }\end{array}$ & $\begin{array}{l}15,2 \mathrm{~b} \\
15,8 \mathrm{ab} \\
17,4 \mathrm{a}\end{array}$ & $\begin{array}{l}18,1 \mathrm{a} \\
18,4 \mathrm{a} \\
19,0 \mathrm{a}\end{array}$ & $\begin{array}{l}0,133 \mathrm{a} \\
0,133 \mathrm{a} \\
0,172 \mathrm{a}\end{array}$ & $\begin{array}{l}21,2 \mathrm{~cd} \\
23,5 \mathrm{~b} \\
26,7 \mathrm{a}\end{array}$ & $\begin{array}{l}28,2 \mathrm{a} \\
28,6 \mathrm{a} \\
29,0 \mathrm{a}\end{array}$ & $\begin{array}{l}0,434 \mathrm{bcd} \\
0,503 \mathrm{abc} \\
0,626 \mathrm{a}\end{array}$ & $\begin{array}{l}24,0 \mathrm{bc} \\
27,3 \mathrm{~b} \\
31,5 \mathrm{a}\end{array}$ & $\begin{array}{l}30,8 \mathrm{ab} \\
32,1 \mathrm{ab} \\
32,4 \mathrm{a}\end{array}$ & $\begin{array}{l}0,612 \mathrm{~cd} \\
0,766 \mathrm{cb} \\
0,963 \mathrm{ab}\end{array}$ \\
\hline $7,0 \mathrm{~m}$ & $\begin{array}{r}1.100 \\
800 \\
400\end{array}$ & $\begin{array}{l}\text { T7 } \\
\text { T8 } \\
\text { T9 }\end{array}$ & $\begin{array}{l}16,2 \mathrm{ab} \\
15,9 \mathrm{ab} \\
16,4 \mathrm{ab}\end{array}$ & $\begin{array}{l}18,8 \mathrm{a} \\
18,1 \mathrm{a} \\
18,1 \mathrm{a}\end{array}$ & $\begin{array}{l}0,150 \mathrm{a} \\
0,140 \mathrm{a} \\
0,141 \mathrm{a}\end{array}$ & $\begin{array}{l}21,3 \mathrm{~cd} \\
23,2 \mathrm{~b} \\
25,8 \mathrm{a}\end{array}$ & $\begin{array}{l}27,5 \mathrm{a} \\
27,0 \mathrm{a} \\
28,1 \mathrm{a}\end{array}$ & $\begin{array}{l}0,409 \mathrm{~cd} \\
0,452 \mathrm{bcd} \\
0,540 \mathrm{ab}\end{array}$ & $\begin{array}{c}24,0 \mathrm{bc} \\
26,8 \mathrm{bc} \\
31,1 \mathrm{a}\end{array}$ & $\begin{array}{l}29,9 \mathrm{ab} \\
30,4 \mathrm{ab} \\
32,4 \mathrm{ab}\end{array}$ & $\begin{array}{l}0,571 \mathrm{~cd} \\
0,684 \mathrm{~cd} \\
0,910 \mathrm{ab}\end{array}$ \\
\hline $\begin{array}{l}\text { Signifi } \\
\text { Raleo } \\
\text { Poda } \\
\text { Poda } x\end{array}$ & Raleo & & $\begin{array}{l}0,004 \\
0,990 \\
0,264\end{array}$ & $\begin{array}{l}0,958 \\
0,566 \\
0,639\end{array}$ & $\begin{array}{l}0,152 \\
0,629 \\
0,394\end{array}$ & $\begin{array}{l}0,000 \\
0,385 \\
0,373\end{array}$ & $\begin{array}{l}0,124 \\
0,177 \\
0,605\end{array}$ & $\begin{array}{l}0,001 \\
0,020 \\
0,613\end{array}$ & $\begin{array}{l}0,001 \\
0,818 \\
0,547\end{array}$ & $\begin{array}{l}0,001 \\
0,256 \\
0,229\end{array}$ & $\begin{array}{l}0,000 \\
0,092 \\
0,238\end{array}$ \\
\hline
\end{tabular}

Nota: Letras diferentes en cada columna indican diferencias significativas (Tukey, p<0,05). Significación: Probabilidades límites en ANOVA con dos factores. Cód: Código del tratamiento, su numeración no corresponde al código empleado en terreno, sólo se utiliza con fines de ordenamiento.

\section{CUADRO 2}

Dimensiones de los 400 árb ha-1 de mayor dap según tratamiento, año de medición y edad de plantación. Size of 400 trees per ha-1 ${ }^{-1}$ of greater dbh according to treatment, year of measurement, and stand age.

\begin{tabular}{|c|c|c|c|c|c|c|c|c|c|c|c|}
\hline \multicolumn{3}{|c|}{ Tratamientos } & \multicolumn{3}{|c|}{ Medición 1995 (6 años) } & \multicolumn{3}{|c|}{ Medición 1999 (10 años) } & \multicolumn{3}{|c|}{ Medición 2003 (14 años) } \\
\hline Poda & Raleo & Cod & $\begin{array}{l}\text { Dap } \\
(\mathrm{cm})\end{array}$ & $\begin{array}{l}\text { Altura } \\
(\mathrm{m})\end{array}$ & $\begin{array}{l}\text { Volumen } \\
\left(\mathrm{m}^{3} \mathrm{arb}^{-1}\right)\end{array}$ & $\begin{array}{l}\text { Dap } \\
(\mathrm{cm})\end{array}$ & $\begin{array}{c}\text { Altura } \\
(\mathrm{m})\end{array}$ & $\begin{array}{l}\text { Volumen } \\
\left(\mathrm{m}^{3} \mathrm{arb}^{-1}\right)\end{array}$ & $\begin{array}{l}\text { Dap } \\
(\mathrm{cm})\end{array}$ & $\begin{array}{c}\text { Altura } \\
(\mathrm{m})\end{array}$ & $\begin{array}{l}\text { Volumen } \\
\left(\mathrm{m}^{3} \mathrm{arb}^{-1}\right)\end{array}$ \\
\hline \multirow[t]{3}{*}{$0 \mathrm{~m}$} & 1.100 & $\mathrm{~T} 1$ & $18,4^{\mathrm{c}}$ & $19,5^{\mathrm{b}, \mathrm{c}}$ & $0,183^{\mathrm{a}, \mathrm{b}}$ & $26,1^{\mathrm{a}}$ & $28,8^{\mathrm{a}}$ & $0,530^{\mathrm{a}}$ & $30,6^{\mathrm{a}}$ & $31,9^{\mathrm{a}}$ & $0,805^{\mathrm{a}}$ \\
\hline & 800 & $\mathrm{~T} 2$ & $18,0^{\mathrm{a}, \mathrm{b}, \mathrm{c}}$ & $18,5^{\mathrm{a}, \mathrm{b}, \mathrm{c}}$ & $0,166^{a, b}$ & $26,6^{\mathrm{a}}$ & $29,5^{\mathrm{a}}$ & $0,593^{\mathrm{a}}$ & $31,5^{\mathrm{a}}$ & $32,9^{\mathrm{a}}$ & $0,887^{a}$ \\
\hline & 400 & $\mathrm{~T} 3$ & $17,0^{\mathrm{a}, \mathrm{b}}$ & $17,8^{\mathrm{a}}$ & $0,148^{\mathrm{a}}$ & $26,9^{\mathrm{a}}$ & $28,9^{\mathrm{a}}$ & $0,597^{\mathrm{a}}$ & $32,3^{\mathrm{a}}$ & $33,6^{\mathrm{a}}$ & $0,987^{\mathrm{a}}$ \\
\hline \multirow[t]{3}{*}{$3,5 \mathrm{~m}$} & 1.100 & $\mathrm{~T} 4$ & $17,8^{\mathrm{a}, \mathrm{b}, \mathrm{c}}$ & $18,7^{\mathrm{a}, \mathrm{b}, \mathrm{c}}$ & $0,178^{a, b}$ & $25,7^{\mathrm{a}}$ & $30,2^{\mathrm{a}}$ & $0,595^{a}$ & $30,4^{\mathrm{a}}$ & $33,7^{\mathrm{a}}$ & $0,904^{\mathrm{a}}$ \\
\hline & 800 & T5 & $17,6^{\mathrm{a}, \mathrm{b}}$ & $19,2^{\mathrm{a}, \mathrm{b}, \mathrm{c}}$ & $0,165^{\mathrm{a}, \mathrm{b}}$ & $27,0^{\mathrm{a}}$ & $30,4^{\mathrm{a}}$ & $0,631^{\mathrm{a}}$ & $32,2^{\mathrm{a}}$ & $34,6^{\mathrm{a}}$ & $0,993^{\mathrm{a}}$ \\
\hline & 400 & T6 & $17,4^{\mathrm{a}, \mathrm{b}}$ & $18,9^{\mathrm{a}, \mathrm{b}, \mathrm{c}}$ & $0,171^{\mathrm{a}, \mathrm{b}}$ & $26,7^{\mathrm{a}}$ & $29,0^{\mathrm{a}}$ & $0,625^{\mathrm{a}}$ & $31,5^{\mathrm{a}}$ & $32,4^{\mathrm{a}}$ & $0,964^{\mathrm{a}}$ \\
\hline \multirow[t]{3}{*}{$7,0 \mathrm{~m}$} & 1.100 & $\mathrm{~T} 7$ & 19,3 & 19,7 & 0,2 & 26 & 30, & 0 , & $30,9^{\mathrm{a}}$ & $33,5^{\mathrm{a}}$ & $0,886^{\mathrm{a}}$ \\
\hline & 800 & $\mathrm{~T} 8$ & $17,8^{\mathrm{a}, \mathrm{b}, \mathrm{c}}$ & $18,9^{\mathrm{a}, \mathrm{b}, \mathrm{c}}$ & 0,173 a,b & $26,3^{\mathrm{a}}$ & $28,6^{\mathrm{a}}$ & $0,562^{\mathrm{a}}$ & $30,9^{\mathrm{a}}$ & $32,3^{\mathrm{a}}$ & $0,858^{\mathrm{a}}$ \\
\hline & 400 & $\mathrm{~T} 9$ & $16,4^{\mathrm{a}}$ & $18,1^{\mathrm{a}, \mathrm{b}}$ & $0,141^{\mathrm{a}}$ & $25,8^{\mathrm{a}}$ & $28,1^{\mathrm{a}}$ & $0,541^{\mathrm{a}}$ & $31,1^{\mathrm{a}}$ & $32,4^{\mathrm{a}}$ & $0,909^{\mathrm{a}}$ \\
\hline \multicolumn{3}{|c|}{ Significación } & & & & & & & & & \\
\hline \multicolumn{3}{|l|}{ Poda } & 0,717 & 0,397 & 0,623 & 0,690 & 0,122 & 0,2 & 0,733 & 0,206 & 0,365 \\
\hline \multicolumn{3}{|c|}{ Raleo } & 0,000 & 0,001 & 0,000 & 0,567 & 0,27 & 0,895 & 0,242 & 0,559 & 0,262 \\
\hline \multicolumn{3}{|c|}{ Poda x Raleo } & 0,035 & 0,026 & 0,084 & 0,573 & 0,199 & 0,551 & 0,711 & 0,027 & 0,644 \\
\hline
\end{tabular}

Nota: Letras diferentes en cada columna indican diferencias significativas (Tukey, p<0,05). Significación: Probabilidades límites en ANOVA con dos factores. Cód: Código del tratamiento, su numeración no corresponde al código empleado en terreno, sólo se utiliza con fines de ordenación. La selección de los árboles de mayor dap se realizó en base a la distribución diamétrica existente en el año 2003. 


\section{CUADRO 3}

Crecimiento en volumen por tratamiento y año de medición para todos los árboles y $\operatorname{los} 400 \mathrm{arb} \mathrm{ha}^{-1}$ de mayor dap.

Volume growth according to treatment and year of measurement for all the trees and for the 400 trees $\mathrm{ha}^{-1}$ of greater $\mathrm{dbh}$.

\begin{tabular}{|c|c|c|c|c|c|c|c|c|c|c|c|}
\hline \multicolumn{3}{|c|}{ Tratamientos } & \multicolumn{3}{|c|}{ Medición 1995 (6 años) } & \multicolumn{3}{|c|}{ Medición 1999 (10 años) } & \multicolumn{3}{|c|}{ Medición 2003 (14 años) } \\
\hline Poda & Raleo & Cod & $\begin{array}{l}\text { Volumen } \\
\left(\mathrm{m}^{3} \mathrm{ha}^{-1}\right)\end{array}$ & $\begin{array}{l}\text { IPA } \\
\left(\mathrm{m}^{3} \mathrm{~h}\right.\end{array}$ & $\begin{array}{r}\text { IMA } \\
\left.\text { año }^{-1}\right)\end{array}$ & $\begin{array}{l}\text { Volumen } \\
\left(\mathrm{m}^{3} \mathrm{ha}^{-1}\right)\end{array}$ & $\begin{array}{l}\text { IPA } \\
\left(\mathrm{m}^{3} \mathrm{ha}^{-1}\right.\end{array}$ & $\begin{array}{l}\text { IMA } \\
\text { año-1) }^{-1}\end{array}$ & $\begin{array}{l}\text { Volumen } \\
\left(\mathrm{m}^{3} \mathrm{ha}^{-1}\right)\end{array}$ & $\begin{array}{l}\text { IPA } \\
\left(m^{3} \mathrm{ha}^{-1}\right.\end{array}$ & $\begin{array}{l}\text { IMA } \\
\left.\text { año }^{-1}\right)\end{array}$ \\
\hline \multicolumn{12}{|c|}{ Todos los árboles } \\
\hline \multirow[t]{3}{*}{$0 \mathrm{~m}$} & 1.100 & $\mathrm{~T} 1$ & 147,8 & 24,6 & 24,6 & 378,3 & 57,6 & 37,8 & 568,5 & 47,6 & 40,6 \\
\hline & 800 & $\mathrm{~T} 2$ & 104,3 & 17,4 & 17,4 & 377,9 & 68,4 & 37,8 & 550,0 & 43,0 & 39,3 \\
\hline & 400 & $\mathrm{~T} 3$ & 62,4 & 10,4 & 10,4 & 258,0 & 48,9 & 25,8 & 426,5 & 42,1 & 30,5 \\
\hline \multirow[t]{3}{*}{$3,5 \mathrm{~m}$} & 1.100 & $\mathrm{~T} 4$ & 146,4 & 24,4 & 24,4 & 486,7 & 85,1 & 48,7 & 680,0 & 48,3 & 48,6 \\
\hline & 800 & $\mathrm{~T} 5$ & 105,4 & 17,6 & 17,6 & 403,6 & 74,6 & 40,4 & 591,0 & 46,9 & 42,2 \\
\hline & 400 & $\mathrm{~T} 6$ & 74,3 & 12,4 & 12,4 & 264,1 & 47,4 & 26,4 & 406,2 & 35,5 & 29,0 \\
\hline \multirow[t]{3}{*}{$7,0 \mathrm{~m}$} & 1.100 & $\mathrm{~T} 7$ & 169,8 & 28,3 & 28,3 & 475,5 & 76,4 & 47,5 & 652,1 & 44,1 & 46,6 \\
\hline & 800 & $\mathrm{~T} 8$ & 110,9 & 18,5 & 18,5 & 367,4 & 64,1 & 36,7 & 555,9 & 47,1 & 39,7 \\
\hline & 400 & T9 & 58,0 & 9,7 & 9,7 & 216,7 & 39,7 & 21,7 & 365,1 & 37,1 & 26,1 \\
\hline \multicolumn{12}{|c|}{ Mayores 400 arb ha-1 } \\
\hline \multirow[t]{3}{*}{$0 \mathrm{~m}$} & 1.100 & $\mathrm{~T} 1$ & 53,3 & 8,9 & 8,9 & 130,3 & 19,3 & 13,0 & 322,8 & 48,1 & 23,1 \\
\hline & 800 & $\mathrm{~T} 2$ & 49,1 & 8,2 & 8,2 & 179,2 & 32,5 & 17,9 & 355,7 & 44,1 & 25,4 \\
\hline & 400 & $\mathrm{~T} 3$ & 62,5 & 10,4 & 10,4 & 257,9 & 48,9 & 25,8 & 426,4 & 42,1 & 30,5 \\
\hline \multirow[t]{3}{*}{$3,5 \mathrm{~m}$} & 1.100 & $\mathrm{~T} 4$ & 53,3 & 8,9 & 8,9 & 174,0 & 30,2 & 17,4 & 362,5 & 47,1 & 25,9 \\
\hline & 800 & $\mathrm{~T} 5$ & 52,0 & 8,7 & 8,7 & 197,7 & 36,4 & 19,8 & 398,2 & 50,1 & 28,4 \\
\hline & 400 & $\mathrm{~T} 6$ & 73,9 & 12,3 & 12,3 & 263,8 & 47,5 & 26,4 & 406,8 & 35,8 & 29,1 \\
\hline \multirow[t]{3}{*}{$7,0 \mathrm{~m}$} & 1.100 & $\mathrm{~T} 7$ & 58,7 & 9,8 & 9,8 & 164,0 & 26,3 & 16,4 & 355,3 & 47,8 & 25,4 \\
\hline & 800 & $\mathrm{~T} 8$ & 56,1 & 9,4 & 9,4 & 181,3 & 31,3 & 18,1 & 344,1 & 40,7 & 24,6 \\
\hline & 400 & $\mathrm{~T} 9$ & 58,1 & 9,7 & 9,7 & 216,9 & 39,7 & 21,7 & 364,5 & 36,9 & 26,0 \\
\hline
\end{tabular}

Al contrario de lo determinado para el total de los árboles de la plantación al año 2003, el IMA de los 400 árb ha-1 de mayor dap tiende a aumentar, para cada nivel de poda, a medida que disminuye la densidad, mientras el IPA tiende a aumentar con la densidad (cuadro 3).

\section{DISCUSION}

Efecto del raleo: El raleo afectó la respuesta de las variables de crecimiento dap, altura y volumen, para cada período de medición, independiente de la intensidad de poda (cuadro 1). El dap medio por árbol del tratamiento de densidad 400 árb ha-1 $^{-1}$ es significativamente mayor a los tratamientos de mayor densidad, especialmente en el control del año 2003. Similares resultados fueron obtenidos por Muñoz y Espinosa (8) y Medhurst y Beadle (16), en estudios con E. nitens. En cuanto a las variables altura y volumen, éstas muestran un comportamiento errático entre tratamientos y años de medición, aunque tienden a ser mayor en los tratamientos con menor densidad residual. Medhurst $e t$ al. (17) encontraron en un experimento de raleo con $E$. nitens, con controles a los 6, 8 y 9 años de edad, que la altura no es afectada por el raleo.

A diferencia de lo encontrado para el total de los árboles de la plantación, no se registraron diferencias significativas en el crecimiento de las variables estudiadas para los 400 árboles por hec- 
tárea de mayor dap (cuadro 2). Lo expuesto implica que estos árboles mantienen una tasa de crecimiento que es independiente del raleo practicado, al menos hasta la fecha del último control.

El volumen de un rodal a una edad dada, está fuertemente afectado por sus componentes dap y altura. En este estudio, los mayores volúmenes acumulados a la edad de 14 años de la plantación, lo alcanzan los tratamientos con mayor densidad (1.100 $\left.\mathrm{arb} \mathrm{ha}^{-1}\right)$, con valores de 568,5, 680,0 y $652,1 \mathrm{~m}^{3} \mathrm{ha}^{-1}$, para T1, T4 y T7, respectivamente, con IMA de 40,6, 48,6 y 46,6 $\mathrm{m}^{3}$ ha $^{-1}$ año-1 $^{-1}$ (cuadro 3). Este último valor es mayor al IPA $(44,1$ $\mathrm{m}^{3} \mathrm{ha}^{-1} \mathrm{año}^{-1}$ ), lo que implica que este tratamiento (T7) ha alcanzado la culminación del incremento medio anual; en la misma situación se encuentra el tratamiento T4 con IPA e IMA de 48,3 y 48,6 $\mathrm{m}^{3} \mathrm{ha}^{-1} \mathrm{año}^{-1}$, respectivamente. La culminación del incremento medio anual es uno de los criterios utilizados para definir la edad de rotación de un rodal (18). Los volúmenes estimados en este estudio se han obtenido hasta un índice de utilización de $5 \mathrm{~cm}$, índice empleado usualmente para regímenes de madera pulpable. En estudios de disponibilidad de madera pulpable de E. nitens, el Infor (3) ha determinado edades de rotación entre 8 y 12 años, edades ligeramente menores a las establecidas en este estudio. Para madera de mayores dimensiones, como la que se utiliza para aserrío y chapas, se requiere de rotaciones mayores. En este estudio, el IPA de los tratamientos T3, T6 y T9, densidad 400 arb ha ${ }^{-1}$, es entre 1,2 y 1,4 veces mayor que el IMA. En estos tratamientos, si se mantiene la tendencia de crecimiento de los últimos 4 años (entre los 10 y 14 años), el IMA se igualaría con el IPA entre los 17 y 18 años de edad. INFOR (19) sugiere seguir esquemas de manejo para madera aserrada y chapas, con rotaciones entre 14 y 22 años, según calidad de sitio y manejo silvícola. Si el supuesto señalado para este estudio se mantiene, se obtendrían rotaciones para madera de mayores dimensiones dentro del rango de edades indicado.

Los IMA obtenidos en este estudio son comparables a los registrados para E. nitens por Barros (20) en la provincia de Valdivia (entre 34,3 y 45,8 $\mathrm{m}^{3} \mathrm{ha}^{-1} \mathrm{año}^{-1}$ a los 12 años), aunque menores a los obtenidos por Prado y Barros (21) en la Octava Región (entre 51 y $77 \mathrm{~m}^{3}$ ha $^{-1}$ año ${ }^{-1}$ a los 15 años). En Tasmania, Australia, se han medido IMA de $27,7 \mathrm{~m}^{3}$ ha $^{-1}$ año $^{-1}$ a la edad de 7 años y densidad de 1010 arb ha- $^{-1}$ (22) y de $26,2 \mathrm{~m}^{3} \mathrm{ha}^{-1} \mathrm{año}^{-1}$ a la edad de 9 años y densidad de 1.031 árb ha-1 $^{-1}$ (17), valores similares a los registrados en este estudio a los 6 años de edad, pero por debajo de los encontrados a la edad de 10 años (cuadro 3).

Efecto de la poda: La poda no tuvo un efecto significativo en el crecimiento de las variables dap, altura y volumen (cuadro 1). Como se ha determinado en numerosos estudios y con diferentes especies, su efecto está relacionado fundamentalmente con la oportunidad e intensidad de aplicación $(23,24,25)$. En este estudio, la poda se practicó cuando la plantación tenía 6 años de edad, en plena fase de competencia intraespecífica, con ramas basales secas o en vías de secarse. Como señalan Mohammed et al. (26), en sitios de alta productividad el cierre de copas en $E$. nitens se produce tempranamente (2,5 años de edad), generando ramas muertas. En este estudio, el tratamiento de poda más intenso $(7,0 \mathrm{~m})$ correspondió, en promedio, sólo a un $38 \%$ de la altura total. Diferentes estudios en E. nitens señalan que podar hasta el $50 \%$ de la altura total, en rodales con densidades iniciales de 800 a 1.200 árb ha $^{-1} \mathrm{y}$ edades de 3 a 5 años, no afecta el incremento en altura y diámetro $(1,6,9,11)$.

\section{CONCLUSIONES}

El raleo incrementa la tasa de crecimiento en diámetro de los árboles. Luego de ocho años de establecido el ensayo, los árboles en las parcelas más intensamente raleadas (400 árb ha ${ }^{-1}$ ) presentan un diámetro significativamente mayor que las no raleadas (1.100 árb ha-1).

La poda no tuvo efecto sobre el crecimiento de los árboles. A la edad de 6 años, oportunidad en que se intervino la plantación, sólo una reducida porción del fuste hasta la altura máxima podada $(7 \mathrm{~m})$ estaba compuesto por ramas verdes.

El volumen por árbol presenta diferencias significativas entre tratamientos. A la edad de 14 años, los mayores volúmenes se alcanzan en los tratamientos más intensamente raleados (400 árb $\mathrm{ha}^{-1}$ ), independientemente de la severidad de poda.

Los valores de dap, altura y volumen medio de los 400 árboles de mayor dap por hectárea de los tratamientos con mayor densidad (1.100 y 800 arb $\left.h^{-1}\right)$, no tienen diferencia significativa con los de menor densidad residual (400 árb ha-1). 
A la edad de 14 años, los árboles de la plantación se encuentran en pleno crecimiento, con incrementos medios anuales en volumen iguales o próximos a la edad de culminación. Se proyecta que antes de los 18 años, todos los tratamientos habrán alcanzado la culminación de su incremento medio anual.

\section{BIBLIOGRAFIA}

(1) PINKARD, E. AND C. BEADLE. Effects of green pruning on growth and stem shape of Eucalyptus nitens (Deane and Maiden) Maiden. New Forests, 1998, vol. 15:, p. $107-126$.

(2) LIGNUM. Eucalyptus nitens: La nueva estrella del sector forestal. Lignum, Fundación Chile. Santiago, Chile, 2003, p. 59-61.

(3) INFOR. Eucalyptus nitens en Chile: Primera monografía. Instituto Forestal. Valdivia. Chile, 2004, Informe Técnico $\mathrm{N}^{\circ} 165,143 \mathrm{p}$.

(4) KUBE, P. AND C. RAYMOND. Breeding to minimize the effects of collapse in Eucalyptus nitens. Technical Report 93 Project A5: Wood quality. Cooperative Research Centre for Sustainable Production Forestry, Hobart, Tasmania, Australia, 2002, 18 p.

(5) SMETHURST, P., C. BAILLIE, M. CHERRY AND G. HOLZ. Fertilizer effects on LAI and growth of four Eucalyptus nitens plantations. Forest Ecology and Management, 2003, vol. 176, p. 531-542.

(6) PINKARD, E., C. BEADLE, N. DAVIDSON AND M. BATTAGLIA.Changes in crown productivity and stem growth of Eucalyptus nitens in response to green pruning. In Eucalyptus plantations: Improving Fibre Yield and Quality. Eds. Potts B M, Borralho N M G, Reid J B, Cromer R N, Tibbits W N, Raymond C A. Proceedings CRCTHF - IUFRO Conference, Feb 19-24, 1995. Hobart, Australia, p. 377-378.

(7) STÖCKLE, M. Efecto inicial de poda y raleo en el crecimiento de un rodal de Eucalyptus nitens de 6 años de edad. Memoria de Título. Ingeniero Forestal. Universidad de Concepción, Facultad de Ciencias Forestales, Departamento Silvicultura. Concepción, Chile, 1996, 26 p.

(8) MUÑOZ, F. y M. ESPINOSA. Efecto de poda y raleo en el crecimiento de un rodal de Eucalyptus nitens. En actas: Realidad y potencial del Eucalipto en Chile: cultivo silvícola y su uso industrial. Silvotecna XII, simposio IUFRO. 26-28 agosto 1999, Concepción, Chile, p. 89-118.

(9) CELHAY, J.A., P. BONNEFOY y F. RIQUELME. Efecto de la intensidad de poda sobre el crecimiento de $E$. nitens. En actas: Realidad y potencial de Eucalipto en Chile: cultivo silvícola y su uso industrial. Silvotecna XII, simposio IUFRO. 26-28 agosto 1999, Concepción, Chile, p. 119-138.

(10) GERRAND, A., W. NEILSEN AND J. MEDHURST. Thinning and pruning eucalyp plantation for sawlog production in Tasmania. Tasforest, 1997, vol. 9, p. 15-34.

(11) NEILSEN, W. AND E. PINKARD. Developing silvicultural regimes for sawlog and veneer production from temperate eucalypt plantations in Tasmania. In: The Future of Eucalypts for wood products. Eds. L Hender- son, G Waugh, G Nolan and P Bennett. Proceedings of IUFRO Conference, 19-24 March 2000, Launceston, Australia, p. 335-348.

(12) CARRASCO, P. y J. MILLÁN. Proyecto de suelos forestales de la VIII Región. Informe final. Universidad de Concepción, Dpto. de Cs. Forestales/Min. de Agricultura. Chillán, Chile., 1990, 152 p.

(13) SANTIBÁÑEZ, F. y J. URIBE. Atlas Agroclimático de Chile, regiones sexta, séptima, octava y novena. Corporación de Fomento. Ministerio de Agricultura. FIA. Santiago, Chile, 1993, 99 p.

(14) STEEL, R. y J. TORRIE. Bioestadística: Principios y procedimientos. McGraw-Hill/Interamericana de México. México, 1988, 622 p.

(15) CMPC. Funciones de volumen para Eucalyptus globulus, nitens, regnans y delegatensis. Area Forestal CMPC. Documento de circulación restringida. Concepción, Chile, 1993, $19 \mathrm{p}$.

(16) MEDHURST, J. y C. BEADLE. Thinning for solid wood products in Eucalyptus nitens plantations. In: The Future of Eucalypts for Wood Products. Eds. L Henderson, G Waugh, G Nolan and P Bennett. Proceedings of IUFRO Conference, 19-24 March 2000, Launceston, Australia, p. 343-348.

(17) MEDHURST, J.L., C.L. BEADLE AND W.A. NEILSEN. Early-age and later-age thinning affects growth, dominance, and intraspecific competition in Eucalyptus nitens plantations. Can. J. For. Res., 2001, vol. 31, p. 187-197.

(18) DANIEL, P.W., U.E. HELMS y F.S. BAKER. Principios de Silvicultura. Segunda ed. McGraw-Hill, México, 1982, 492 p.

(19) INFOR. Eucalyptus nitens en Chile: Economía y mercado. Instituto Forestal. Valdivia, Chile, 2004, Informe Técnico $\mathrm{N}^{\circ} 166,80 \mathrm{p}$.

(20) BARROS, S. Crecimiento juvenil de especies y procedencias de Eucalyptus -Los Copihues- provincia de Valdivia, X región. Actas Simposio: Los Eucaliptos en el desarrollo forestal de Chile. Eds. S Barros, J A Prado y C Alvear. 24-26 Nov 1993. Pucón, Chile, p. 207-226.

(21) PRADO, J.A. y S. BARROS. Eucalyptus. Principios de silvicultura y manejo. Corporación de Fomento de la Producción. Santiago, Chile, 1991, 199 p.

(22) PINKARD, E. AND W. A. NIELSEN. Crown and stand characteristics of Eucalyptus nitens in response to initial spacing: implications for thinning. Forest Ecology and Management, 2003, vol. 172, p. 215-227.

(23) SUTTON, W. Pino radiata: sus excepcionales perspectivas en el comercio mundial de productos forestales. Fundación Chile, Depto. Forestal. Santiago, Chile, 1985, Publicación Técnica $\mathrm{N}^{\circ} 17,32 \mathrm{p}$.

(24) BARRET, J. Pruning of ponderosa pine. Effect on growth. U.S.D.A. For. Serv. Res. Paper PNW68. Portland, Oregon, 1968, 9 p.

(25) MACLAREN, J. Radiata pine Grower's Manual. New Zealand Forest Research Institute. FRI. Rotorua, New Zealand, 1993, Bulletin 184, 140 p.

(26) MOHAMMED, C., K. BARRY, M. BATTAGLIA, C. BEADLE, A. EYLES, A. MOLLON AND E. PINKAR. Pruning-associated stem defects in plantation E. nitens and E. globulus grown for sawlog and veneer in Tasmania, Australia. In: The Future of Eucalypts for wood products. Eds. L Henderson, G Waugh, G Nolan and P Bennett. Proceedings of IUFRO Conference, 19-24 March 2000, Launceston, Australia, p. 357-364. 